\title{
Marital Satisfaction \\ Among Spouses of Male Patients with Alcohol Dependence Syndrome
}

\author{
Humayoon Akbar, Sudhakar S, Shanthi B, Khadeja Bi, Jayaseelan R, Nithya HM
}

- Department of Psychiatry, Chengalpattu Medical College

\section{ABSTRACT}

\begin{abstract}
BACKGROUND
One third of Indians consume alcohol and there is an alarming annual increase in alcohol consumption. Majority of spouses of alcohol dependent males are also the primary care-takers of their husbands and are at risk of domestic violence. Marital dissatisfaction could reduce their involvement in getting their spouses treated for alcohol dependence. Our aim was to study the marital satisfaction among the spouses of patients with alcohol dependence.
\end{abstract}

\section{METHODOLOGY}

This study was conducted on spouses of male patients with alcohol dependence syndrome attending the psychiatry department of a tertiary care medical college hospital in Kanchipuram District in Tamilnadu. 200 consecutive male patients with alcohol dependence syndrome using the ICD 10 criteria were recruited. Severity of alcohol dependence was assessed using Short Alcohol Dependence Data (SADD). Marital satisfaction among the spouses was assessed using ENRICH Marital Satisfaction (EMS) Scale. Analysis of the data was done using Statistical Package for the Social Sciences (SPSS) version 22.0.

\section{RESULTS}

The mean age of alcohol dependent men and their spouses were $43.05 \pm 9.39$ and $37.58 \pm 8.86$ years respectively. Most of the spouses (83.5\%) had done their primary education only and 50\% were house-wives (unemployed). Majority of men earned between Rs.5000 to 10,000 and most of them were unskilled workers belonging to the lower socio-economic group in the rural areas. The mean SADD and EMS scores were $25.05 \pm 8.891$ and $31.76 \pm$ 14.45 respectively. $74 \%$ of men in our study population were highly dependent on alcohol. Amongst the spouses of men with severe dependence $68.9 \%$ reported moderate and $28.3 \%$ reported low marital satisfaction.

\section{DISCUSSION \& CONCLUSION}

Majority of alcohol dependent men suffered from severe dependence. An inverse relationship between marital satisfaction scores and severity of alcohol dependence was observed. Alcohol dependence and its severity was noted to have an adverse impact on marital satisfaction among spouses of the dependent patients

\section{Corresponding author:}

Dr. Sudhakar S,

Department of Psychiatry,

Chengalpattu Medical College, Chengalpattu 603001.

Email: sudhakarpsychiatrist@yahoo.com

KEY WORDS: Alcohol Dependence, Spouse, Marital Satisfaction. 


\section{INTRODUCTION}

One third of Indians consume alcohol and there is an alarming annual increase in alcohol consumption. ${ }^{(1)}$ A recent WHO report also states, there is an annual increase in global alcohol consumption. (2) Heavy drinking is more common in men, in lower socioeconomic groups, in those with lower educational levels and in the young. ${ }^{(3)}$ Majority of spouses of alcohol dependent males are also the primary care-takers of their husbands and are at risk of domestic violence. (4) Marital dissatisfaction and maladjustment reduce their involvement in getting their husbands treated for alcohol dependence. ${ }^{(5)}$

\section{MATERIAL \& METHODS}

This cross - sectional study was conducted on spouses of two hundred consecutive males with alcohol dependence syndrome attending the psychiatry department of a tertiary care medical college hospital in Kanchipuram District in Tamilnadu after obtaining the institutional ethical committee approval and written informed consent from participants. Males ( $\geq 18$ years of age) fulfilling the diagnostic criteria for alcohol dependence according to ICD 10 and their spouses who offered consent to participate in the study were included. Spouses with major physical illness or unable to accompany the husband with alcohol dependence syndrome to the psychiatry department and those not offering consent to participate in the study were excluded. Socio-demographic profile was obtained using a semi-structured proforma. Severity of alcohol dependence in male alcoholics was assessed using Short- form Alcohol Dependence Data (SADD). Marital satisfaction among spouses was assessed using ENRICH Marital Satisfaction (EMS) Scale.

\section{STATISTICAL ANALYSIS}

Analysis was done using Statistical Package for the Social Sciences (SPSS) version 22.0. Descriptive statistics were analysed and expressed as mean and standard deviation for numerical variables and frequencies for nominal and ordinal variables. The relationship between continuous variables was evaluated using the Student's $t$-test. Statistical significance was considered when $\mathrm{p}<0.05$. Co-relation between the variables was analysed using Spear-man's co-relation co-efficient test.

\section{RESULTS}

\section{SOCIO-DEMOGRAPHIC PROFILE}

The age of the alcohol dependent men ranged from 21 to 67 years with mean age of $43.05 \pm 9.39$ years while the age of spouses of alcohol dependent men ranged from 19 to 63 years with a mean of $37.58 \pm 8.86$ years. Chart 1 depicts the age distribution among alcohol dependent male patients and their wives. Frequency was highest in 31 to 40 years age group in both husband and wives. They constituted around $36 \%$ and $43 \%$ of the study population. Chart 2 depicts the educational status of alcohol dependent men and their spouses. Majority of the participants $(83.5 \%$ of men and $51 \%$ of wives) had only attended primary school. Around $11 \%$ of men and $35.5 \%$ of women were illiterate. Only $1 \%$ of the study participants had a university degree.

Chart 3 depicts the employment status of alcohol dependent men and their spouses. Majority of men $(83.5 \%)$ were semi-skilled workers while most of the wives $(50.5 \%)$ were unemployed. Chart 4 shows the income earned by the alcohol dependent men and their spouses per month. $98 \%$ of men earned between Rs. 5000 to 10,000 while most of the women were housewives and the families largely belonged to the lower socioeconomic status and residing rural areas. Chart 5 depicts the general health condition and presence of co-morbid physical illness among spouses of alcohol dependent men during the period of the study. $73.5 \%$ of spouses did not suffer from any physical illness, $10.5 \%$ suffered from asthma, 9.5\% had diabetes mellitus and $6.5 \%$ had a history of seizures.

\section{Chart 1: Age distribution among alcohol dependent male patients and their spouses}

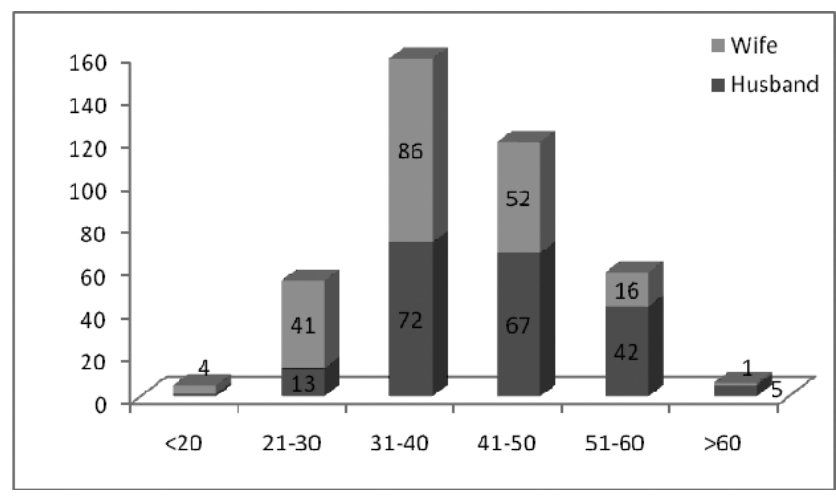


Chart 2: Educational status among alcohol dependent men and their spouses

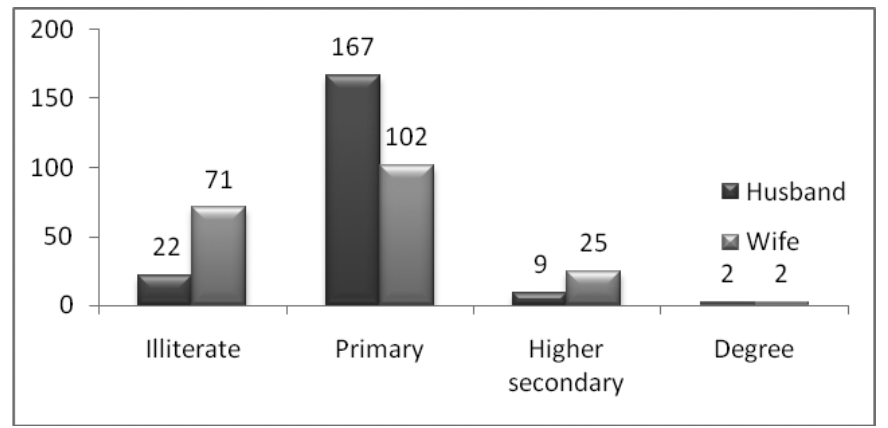

Chart 3: Employment status among alcohol dependent men and their spouses

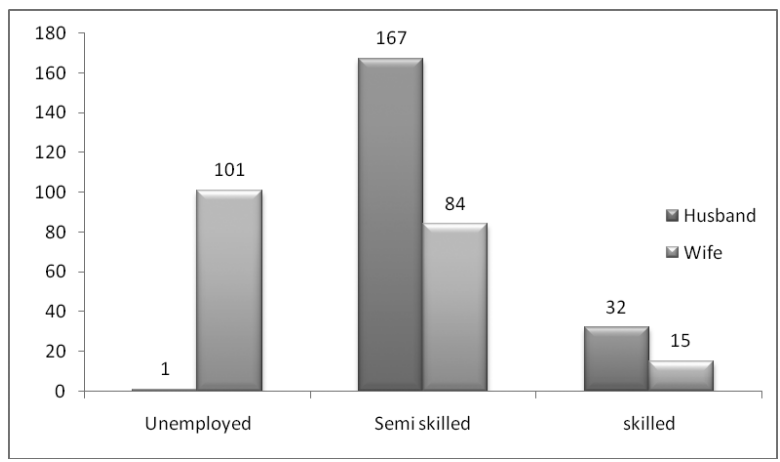

Chart 4: Income of alcohol dependent men and their spouses

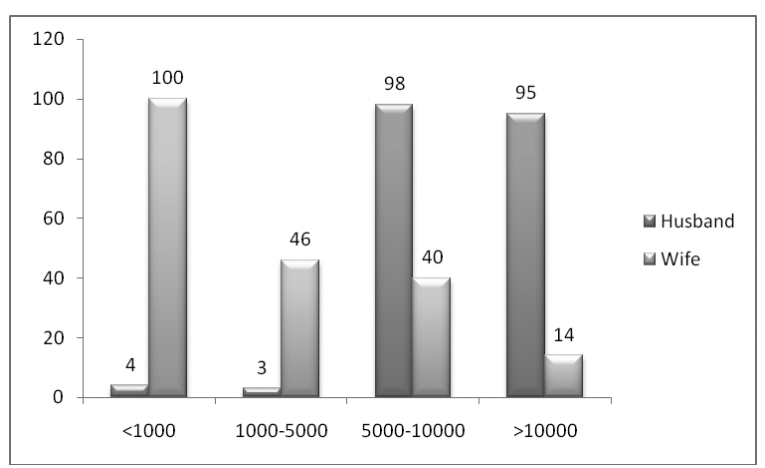

Chart 5: General health condition among spouses of alcohol dependent males

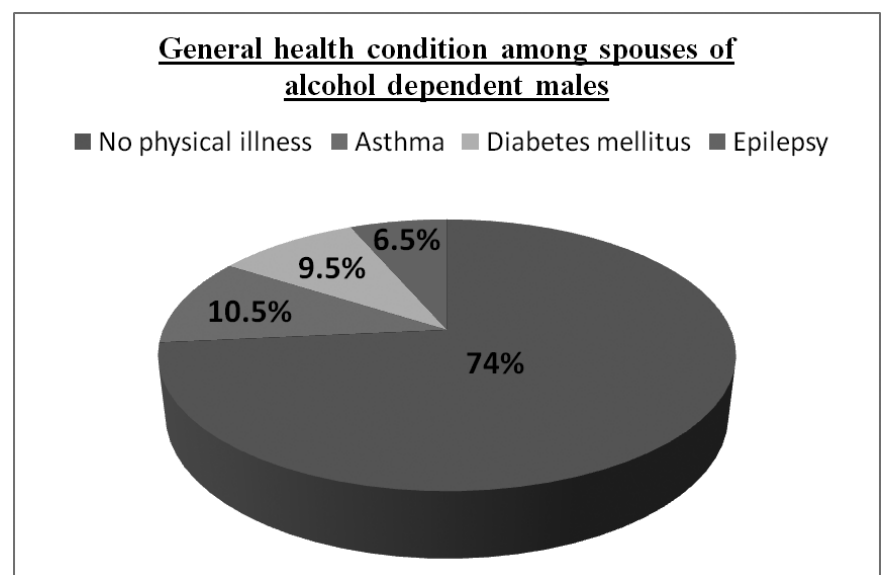




\section{SCALES \& SCORING}

Table 2 classifies the men according to their levels of alcohol dependence based on SADD scores. $74 \%$ of men in our study population were highly dependent on alcohol while $13 \%$ were found to have low and medium dependence. Majority of wives had their EMS scores in the moderate $(21-40)$ and lower $(\leq 20)$ zones of the scale indicating lesser degree of marital satisfaction in spouses of men with alcohol dependence syndrome. Table 3 depicts the frequency distribution of SADD versus EMS scores when classified.
All men with low dependence had all their spouses in the high marital satisfaction range. Majority of spouses (13\%) with high marital satisfaction had their husbands who were with low alcohol dependence. Amongst wives of men with severe dependence $68.9 \%$ reported moderate and $28.3 \%$ reported low marital satisfaction. Spearman's correlation coefficient obtained a moderate negative correlation coefficient of -0.582 between ENRICH Marital Satisfaction and SADD score which was significant with p-value of 0.0001 (Table 4).

Table 1: Short Alcohol Dependence Data (SADD) \& Enrich Marital Satisfaction (EMS)

\begin{tabular}{|l|c|c|c|}
\hline Scores & $\begin{array}{c}\text { RANGE } \\
(\mathrm{n}=200)\end{array}$ & $\begin{array}{c}\text { MEAN } \pm \text { SD } \\
(\mathrm{n}=200)\end{array}$ & $\begin{array}{c}\text { MEDIAN } \\
(\mathrm{n}=200)\end{array}$ \\
\hline Total SADD $(1-45)$ & $8-44$ & $25.05 \pm 8.891$ & 29 \\
\hline EMS $(12.96-60)$ & $14-60$ & $31.76 \pm 14.45$ & 27.5 \\
\hline
\end{tabular}

Table 2: Level of alcohol dependence based on SADD Score

\begin{tabular}{|l|c|c|}
\hline Level of dependence based on SADD Score & Frequency & Percent \\
\hline Low dependence (1-9) & 26 & 13 \\
\hline Medium dependence (10-19) & 26 & 13 \\
\hline High dependence (20-45) & 148 & 74 \\
\hline Total & 200 & 100 \\
\hline
\end{tabular}

Table 3: Frequency distribution of SADD versus EMS scores when classified

\begin{tabular}{|l|c|c|c|}
\hline & $\begin{array}{c}\text { Low satisfaction score } \\
(\leq 20) \text { in EMS }\end{array}$ & $\begin{array}{c}\text { Moderate satisfaction score } \\
(21-40) \text { in EMS }\end{array}$ & $\begin{array}{c}\text { High satisfaction score } \\
(41-60) \text { in EMS }\end{array}$ \\
\hline Frequency & Number & Number & Number \\
\hline $\begin{array}{l}\text { Low dependence score } \\
(1-9) \text { in SADD }(\%)\end{array}$ & 0 & 0 & $26(100)$ \\
\hline $\begin{array}{l}\text { Medium dependence score } \\
(10-19) \text { in SADD (\%) }\end{array}$ & $02(7.6)$ & $07(26.9)$ & $17(65.3)$ \\
\hline $\begin{array}{l}\text { Severe dependence score } \\
(20-45) \text { in SADD }(\%)\end{array}$ & $42(28.3)$ & $99(68.9)$ & $07(04.7)$ \\
\hline TOTAL & 44 & 106 & 50 \\
\hline
\end{tabular}

Table 4: Spearman's correlation coefficient for ENRICH marital satisfaction scores SADD score

\begin{tabular}{|l|c|c|c|}
\hline Dependent score & Independent scores & Co-relation & P value \\
\hline EMS score & Total SADD score & -0.582 & 0.0001 \\
\hline
\end{tabular}




\section{DISCUSSION:}

In our study, the age distribution among alcohol dependent male patients and their wives was highest in 31 to 40 years age group. They constituted around $36 \%$ and $43 \%$ of the study population. This result is similar in a study done by Dandu et al where majority of wives of alcohol dependent males were in age group between 31 to 40 years. ${ }^{(4)}$ Sarkar et al in their study from eastern India also found that majority of the alcohol dependent cases were from the age group of 20 - 49 years (85\%). (5) A study from Bangalore, India found preponderance of the alcoholic cases in the age range of 20 - 50 years. (6) Another study by Soni et al also had the mean age of alcohol dependent men as $32 \pm 7.2$ and that of controls $34 \pm 5.8 .^{(7)}$

Observations from our study revealed that majority comprising, $83.5 \%$ of both alcohol dependent men and their spouse had completed only their primary schooling. Similar results were noted by Ghosh P et al where $60.86 \%$ of spouses of alcohol dependent men were educated only up to the primary level. ${ }^{(8)}$ Studies done by Sarkar et al and Soni et al observed that majority of alcohol dependent men had completed their primary schooling only. ${ }^{(5,7)}$ Around $50 \%$ of wives of alcohol dependent men were home makers (unemployed) in our study. According to Ghosh P et al and Bagul, 78.26\% and $68.3 \%$ respectively of spouses of alcohol dependent men were unemployed in their study. ${ }^{(8,9)}$ In our study, majority of men earned between Rs.5000 to 10,000 and most of them were unskilled workers. This is similar to previous studies where most of the alcohol dependent men were unskilled workers (37.68 \%, $76 \%$ and $55 \%$ respectively). . $^{(7,9)}$

In our study, on administering SADD to measure the severity of alcohol dependence, mean score obtained was $25.05 \pm 8.891$, indicating significant dependence. $13 \%$ of patients had low, $13 \%$ had medium and $74 \%$ had high dependence in our study. This is similar to a study done by Soni et al where on administering SADD, the mean score found was $24.86 \pm 10.1$, indicating significant dependence in alcohol dependent men, 46 $\%$ of them were found to be moderately dependent on alcohol consumption, while $42 \%$ were severely dependent according to SADD scores. ${ }^{(7)}$ Also, another study done by Bagul et al where SADD scores were administered, SADD scores ranged from 4-45 and mean score was 20.35. Severity of alcohol dependence on SADD in males with alcohol dependence suggested significant dependence. Almost equal numbers of alcohol dependent males were found in the moderate and high dependence range. ${ }^{(9)}$

The scores on ENRICH marital satisfaction scale (EMS) were in range of 14 to 60 . The mean EMS score was 31.76. Majority of the scores were in the lower range indicating lesser degree of marital satisfaction. All wives of those with low dependence scores reported higher satisfaction. This is similar to a study done by Kishore et al where the marital satisfaction scores were in the range from 1 - 57 and almost all of the spouses had scores at the lower end of the scale indicating lower degree of marital dissatisfaction (mean $26.7 \pm 12.8$ ). Also, marital satisfaction scores decreased as severity of dependence increased. Hence he concluded that the severity of alcohol dependence obtained from SADD scores was strongly associated with marital dissatisfaction in the spouses. ${ }^{(10)}$

In a study done by Vimala et al, marital satisfaction was negatively correlated with severity of alcohol dependence thereby concluding that spouses of alcohol dependent men had lower levels of satisfaction. ${ }^{(11)}$

\section{CONCLUSION:}

In our study, among all wives who reported lower marital satisfaction, $95 \%$ of husbands had high dependence scores. This study concludes that alcohol dependence in men can significantly impact on the quality of marital life among the spouses.

\section{LIMITATIONS OF THE STUDY}

This is a hospital - based study, at risk of recruitment bias. Marital satisfaction was based on self-report only. The study only had a cross sectional design and follow up of the participants would have been useful to find out if successful treatment improves marital satisfaction. More research involving a larger community - based sample is required to corroborate the findings of our study.

\section{REFERENCES}

1 Ray R. The extent, pattern and trends of drug abuse in India: National Survey. 2004, Ministry of Social Justice and Empowerment and United Nations Office on Drugs and Crime.

2 WHO Expert Committee Report on Problems Related to Alcohol Consumption. WHO Technical Report Series 944, 2007: 9 - 23.

Available at http://www.who.int/substance_abuse/expert_committee_alcohol_trs944.pdf.

3 Abhijit Nadkarni, Helen A. Weiss, Bhargav Bhat, Vikram Patel. Trajectories of alcohol use disorders and their differential impact: A population- based cohort study in Goa, India. Alcohol and Alcoholism, 2017, 52(5): 557-563. 
4 Aruna Dandu, S. Bharathi, Shankar Reddy Dudala. Psychiatric morbidity in spouses of patients with alcohol related disorders. J Family Med Prim Care. 2017, 6(2): 305-310.

5 Sarkar S, Patra BN, Kattimani S. Substance use disorder and the family: An Indian prospective. Med J D Y Patil Univ. 2016, 9: 7-13.

6 Gururaj G, Girish N, Benegal V. Burden \& Socio-Economic Impact of Alcohol-Bangalore Study. New Delhi, Regional Office for South-East Asia, 2006

7 Ruchi Soni, Ritesh Upadhyay, Mahendra Jain. Psychiatric morbidity, Quality of life and Marital Satisfaction among spouses of men with alcohol dependence syndrome: A study from North India. IOSR Journal of Dental and Medical Sciences. 2016, 15(11): 70-76.

8 Prosenjit Ghosh, Robin Victor, Himabrata Das, Shruti Sharma. Psychiatric morbidity and marital satisfaction: an empirical study on spouse of alcohol dependent cases in Barak valley, North-East India. International Journal of Research in Medical Sciences. 2017, 5(4): 1600-1606.

9 Koustubh R. Bagul, Shrikant B. Deshmukh, Monika K. Bagu, Parth S. Deshmukh. Psychiatric morbidity and marital quality among wives of patients with alcohol dependence syndromes. Journal of Evidence Based Medicine and Healthcare. 2015, 2(22): 3284-3295.

10 Kishor M, Pandit LV, Raguram R. Psychiatric morbidity and marital satisfaction among spouses of men with alcohol dependence. Indian J Psychiatry. 2013, 55(4): 360-365.

11 Vimala G. Marital Satisfaction and Burden among Wives of men with Alcohol Dependence Attending at psychiatric OPD of Prayars Rural Hospital. International Journal of Advances in Nursing Management. 2016, 4(4): 361-364. 